\title{
Characterization of heat-moisture treated Dioscorea alata purpurea flour: impact of moisture level
}

\begin{abstract}
Purpose: Edible bird's nest (EBN) has been considered as one of the nutritious foods and was also claimed to aid in digestion problems. Potential prebiotic of the EBN for gut health by the presence of glycan within the complex structure of the EBN glycoprotein to date has not been reported. The gut health can contribute to the overall consumers' health in the improvement of the gut beneficial bacterial growth. In this study, the potential prebiotic of the EBN was conducted using a simulation of in vitro human colon model system.
\end{abstract}

Methods: The EBN-extracted glycan and EBN glycoprotein (crude sample) were digested using in vitro oral, gastric and duodenal model system. Prebiotic activities of the undigested EBN glycan and EBN glycopeptide compounds were studied with the fructooligosaccharide as a positive control, using inoculum of $10 \%(\mathrm{w} / \mathrm{v})$ faecal bacteria in the in vitro fermentation system.

Result: The fermentation of EBN glycan and EBN glycopeptide had shown significant increases of the gut beneficial bacteria and was comparable with fructooligosaccharide fermentation, with each sample presented different profiles of bacterial growth. The fermentation of EBN glycan and EBN glycopeptide demonstrated an increase in the total short-chain fatty acid production, particularly acetate, propionate and butyrate.

Conclusion: These findings suggested that the EBN can be functioned as a natural prebiotic upon consumption, thus providing a potential as prebiotic ingredients.

Keyword: Carbohydrate leaching; Dioscorea alata purpurea; Gelatinization temperatures; Heat-moisture treatment; Swelling 